\title{
Numerical and experimental investigation of bump foil mechanical behaviour
}

\author{
Larsen, Jon Steffen; Cerda Varela, Alejandro Javier; Santos, Ilmar
}

Published in:

Tribology International

Link to article, DOI:

10.1016/j.triboint.2014.02.004

Publication date:

2014

Link back to DTU Orbit

Citation (APA):

Larsen, J. S., Cerda Varela, A. J., \& Santos, I. (2014). Numerical and experimental investigation of bump foil mechanical behaviour. Tribology International, 74, 46-56. https://doi.org/10.1016/j.triboint.2014.02.004

\section{General rights}

Copyright and moral rights for the publications made accessible in the public portal are retained by the authors and/or other copyright owners and it is a condition of accessing publications that users recognise and abide by the legal requirements associated with these rights.

- Users may download and print one copy of any publication from the public portal for the purpose of private study or research.

- You may not further distribute the material or use it for any profit-making activity or commercial gain

- You may freely distribute the URL identifying the publication in the public portal

If you believe that this document breaches copyright please contact us providing details, and we will remove access to the work immediately and investigate your claim. 


\section{Author's Accepted Manuscript}

Numerical and experimental investigation of bump foil mechanical behaviour

Jon S. Larsen, Alejandro C. Varela, Ilmar F. Santos

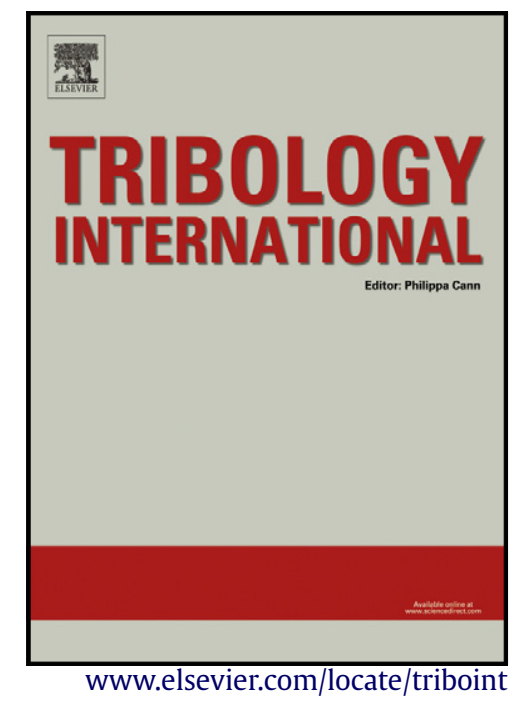

PII:

S0301-679X(14)00055-3

DOI: $\quad$ http://dx.doi.org/10.1016/j.triboint.2014.02.004

Reference: JTRI3249

To appear in: Tribology International

Received date: 26 November 2013

Revised date: 4 February 2014

Accepted date: 10 February 2014

Cite this article as: Jon S. Larsen, Alejandro C. Varela, Ilmar F. Santos, Numerical and experimental investigation of bump foil mechanical behaviour, Tribology International, http://dx.doi.org/10.1016/j.triboint.2014.02.004

This is a PDF file of an unedited manuscript that has been accepted for publication. As a service to our customers we are providing this early version of the manuscript. The manuscript will undergo copyediting, typesetting, and review of the resulting galley proof before it is published in its final citable form. Please note that during the production process errors may be discovered which could affect the content, and all legal disclaimers that apply to the journal pertain. 


\title{
Numerical and experimental investigation of bump foil mechanical behaviour
}

\author{
Jon S. Larsen ${ }^{\mathrm{a}, \mathrm{b}}$, Alejandro C. Varela ${ }^{\mathrm{a}}$, Ilmar F. Santos ${ }^{\mathrm{a}}$ \\ ${ }^{a}$ Department of Mechanical Engineering, Technical University of Denmark, 2800 Kgs. Lyngby, Denmark \\ ${ }^{b}$ Siemens A/S - Aeration Competence Centre, 3000 Helsing $\phi r$, Denmark
}

\begin{abstract}
Corrugated foils are utilized in air foil bearings to introduce compliance and damping thus accurate mathematical predictions are important. A corrugated foil behaviour is investigated experimentally as well as theoretically. The experimental investigation is performed by compressing the foil, between two parallel surfaces, both statically and dynamically to obtain hysteresis curves. The theoretical analysis is based on a two dimensional quasi static FE model, including geometrical non-linearities and Coulomb friction in the contact points and neglects the foil mass. A method for implementing the friction is suggested. Hysteresis curves obtained via the FE model are compared to the experimental results obtained. Good agreement is observed in the low frequency range and discrepancies for higher frequencies are thoroughly discussed.
\end{abstract}

Keywords: gas foil bearing, foil structure, finite element method, friction, hysteresis

\section{Introduction}

The static and dynamic characteristics of compliant foil bearings are determined by the behaviour of the fluid film and a flexible element underneath the bearing surface altering its compliance. Several configurations are possible to obtain compliance, being the usage of corrugated bump foils one of the most widely used. The addition of these compliant elements into the design enables to introduce additional damping to the one generated in the fluid film. The increase of the energy dissipation is obtained due to the sliding friction forces, generated as the bearing surface deforms and induces displacements in the foil layers. However, the mechanism for obtaining the additional damping characteristics exhibits highly non-linear behaviour, which introduces significant complexities considering the obtention of an acceptable level of predictability for this bearing design.

The challenges related to the technology have generated a significant number of publications, dealing with the theoretical modelling and experimental testing of bump foil bearings. The presentation given here tries to follow a chronological progression, and focuses on the

Email addresses: josla@mek.dtu.dk (Jon S. Larsen), acer@mek.dtu.dk (Alejandro C. Varela), if s@mek.dtu.dk (Ilmar F. Santos)

Preprint submitted to Tribology International ones that have influenced the development of the work presented in this article. Namely, the isolated static and dynamic characterization of the corrugated foil structure by neglecting the fluid film effects.

$\mathrm{Ku}$ and Heshmat [1, 2, 3] presented an analytical mathematical bump foil model based on the work of Walowit [4]. The model considered a circular bearing and took into account the effect of the pad location. The model provided predictions for stiffness, hysteresis and equivalent viscous damping. Non-linear stiffness behavior was attributed to the geometrical effects of having a circular journal loading the foils. They predicted that the dynamic coefficients were anisotropic and highly non-linear and that the stiffness and damping was dependant on the pad angle. Bump stiffness under different load distributions along the bump strip was also investigated [1] and the theoretical prediction followed the trend of earlier experimental data, regarding the higher stiffness of the bumps located at the fixed end compared to those closer to the free end. Lower friction coefficients was found to make bumps softer, whereas an increment in friction increased the stiffness and could result in pinned bump ends for the bumps close to the fixed end.

Experimental results of hysteresis curves for bump strips deformed between two straight surfaces was presented in [5]. One of the surfaces featured a pivot to

February 18, 2014 
enable tilting motion, in order to obtain different load distributions over the foils. The effect of pivot location and different surface coatings was investigated and the bump deflections were recorded using an optical tracking system. 'Local' stiffness and damping were identified and found to be dependant on amplitude and load.

Peng and Carpino [6] were among the first ones to couple the bump structure with the fluid film in a mathematical model. Coulomb friction forces and bump flexibility were included by means of an equivalent continuous friction force and a spring constant. Stiffness and damping coefficients were calculated using the coupled model. No isolated validation of the foil structural model was included in this work.

$\mathrm{Ku}$ and Heshmat [7, 8] performed a experimental investigation of the dynamic behaviour of a compliant foil bearing and compared the results to the mathematical model presented in [1, 2, 3]. Agreement between the theoretical and experimental results was reasonably good. The results showed that the cross coupling stiffness and damping are negligible and that the direct terms decrease with increasing dynamic amplitude. An increase of the excitation frequency was found to decrease the equivalent viscous damping and to increase the stiffness.

Similar experiments were performed by Rubio and San Andres [9, 10]. These authors compared the experimental results to the ones obtained using a simplified mathematical model, in which the bump foil contribution was represented by simple elastic springs. The stiffness of these springs was calculated by the analytical expression of Iordanoff [11]. Furthermore, the equivalent damping was determined experimentally, for a given bump geometry, by assuming a one DOF system to which the experimental data was fitted $[12,13]$. This method is based on the assumption of harmonic oscillations which can be hard to obtain in an experimental set-up. Temperature effects was also investigated [12] and found to be negligible. The dry friction coefficient was found to be nearly constant with the excitation frequency but dependent on the load amplitudes. The obtained friction coefficient values varied between 0.05 to 0.2 .

An NDOF discrete bump formulation model including the effect of Coulomb friction was presented by Le Lez and Arghir [14, 15]. The foil structural model was composed of simple spring elements with elementary stiffness given by analytical expressions. The results were compared to a detailed finite element (FE) model based on a commercial software as well as experimental data [14] with good agreement. Furthermore, the calculated stiffness was compared to the simple foil flexibil- ity given by Walowit [4] and implemented in the simple elastic foundation model by Heshmat $[16,17]$. The updated results were found to be significantly stiffer than the reference ones, due to the inclusion of the dry friction effect.

Lee et al. [18] presented a mathematical model incorporating both the fluid film pressure field described by Reynolds equation and the structural dynamics of the foil structure. The solution was based on FEM analysis, and it was performed using a time domain integration routine. An algorithm to deal with the stick slip phenomenon related to friction forces was incorporated as well. A parametric study was performed, and hysteresis loops were presented for the bearings running under steady state conditions. The dissipated energy for the individual bumps were calculated at a given unbalance. The study indicated that optimum values of bump stiffness and friction coefficients exist with regard to minimizing the resonance vibration response of a rotor mounted on foil bearings.

Zywica [19, 20] simulated the top foil structure using commercial FE programs and compared to results previously published in [10]. This structural model was applied in a complex model [21] taking into account the fluid film pressure by solving the Reynolds equation. The study was of purely theoretical nature.

Considering the literature background given here, this article is focused on the global, quasi-static and dynamic, behaviour of a bump foil strip and the local behaviour in its individual sliding contact points. This is achieved through mathematical modelling and experimental observations. The study focuses on a bump foil strip, pressed between two parallel surfaces. This original approach enables a direct comparison between experimental and theoretical results. The structural mathematical model is based on the finite element method (FEM) and the virtual work principle, applied to the studied foil geometry. Hence, the entire bump foil strip is modelled explicitly, using non-linear large deformation theory. The Coulomb friction forces are modelled using an original approach, based on equivalent nonlinear springs located in the contact points between the bump foils and the mating surfaces, acting in the direction of the bump longitudinal displacement. The model is set up so that the correct direction of the friction force at each contact point is directly obtained, eliminating the need for updating the forcing term. It was implemented in a dedicated computer program and the theoretical results, concerning the quasi-static behaviour of the bump foils, are compared against results both from the literature, but mainly against the experimental data obtained in a test rig designed and built for this purpose. 


\section{Theoretical Model}

A theoretical model of the foil structure has been developed and implemented. It takes into account large bump foil deflections and Coulomb sliding friction. The model is based on a non-linear FE procedure following the iterative Newton-Raphson (NR) approach derived in Appendix A. The foil structure is discretized following the virtual work principle (VWP) and a bilinear quadrilateral $(\mathrm{Q} 4)$ iso-parametric plain strain element and the Green-Lagrange strain measure for large displacements are implemented.

The mathematical model is quantified in terms of the residual vector $\{R\}$ and the tangent matrix $\left[K_{t}\right]$ which, combined with the NR approach, solves for the displacement vector $\{D\}$. With the exception of the friction elements, the derivation of these quantities are thoroughly described in the literature $[22,23]$ and for the sake of briefness omitted here.

\subsection{Modelling Friction}

The reaction force between two contacting bodies can be decomposed in two forces; the normal force $F_{n}$ and the friction force $F_{\mu}$. If Coulomb friction law is assumed and the static and dynamic friction coefficients are equal, the friction force $F_{\mu}$ can be written as:

$$
F_{\mu}= \begin{cases}F_{n} \mu, & \text { if } \dot{x}_{r}<0 \\ -F_{n} \mu, & \text { if } \dot{x}_{r}>0\end{cases}
$$

and

$$
-F_{n} \mu \leq F_{\mu} \leq F_{n} \mu, \quad \text { if } \dot{x}_{r}=0
$$

where $\dot{x}_{r}$ is the relative sliding velocity in the contact point and $\mu$ is the coefficient of friction. Consequently, the friction force $F_{\mu}$ is a function of the sliding velocity $\dot{x}$ and is continuous but non-linear. It could be included in the FE model as a nodal load, illustrated in Fig. 1a, in which case, the magnitude and sign of the force would be unknown unless an iterative procedure with checks for sliding direction i.e. sign of $\dot{x}_{r}$ and updates of the nodal reaction force $F_{n}$ were introduced.

An alternative method is to add a spring in the point of contact as illustrated in Fig. 1b. The first thing to note when considering this method is, that the problem of determining the sign of the force $F_{\mu}$ is eliminated since the reaction force of the spring $k$ will automatically be in the opposite direction of the motion $\dot{x}_{r}$. The magnitude of the reaction force would not be constant if the spring $k$ is linear though. Then it would increase linearly with the movement of the contact point, which is obviously a)

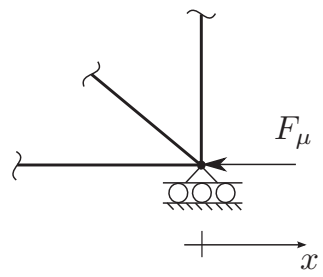

b)

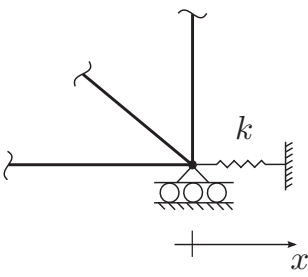

Figure 1: (a) Modelling friction with a nodal load $F_{\mu}$. (b) Modelling friction by use of a non-linear spring $k(\varepsilon)$

wrong. However, by choosing the stiffness $k$ to be nonlinear and softening, the reaction force versus deflection can be made constant and fulfilling (1). Choosing a proper stiffness function for $k$, can even eliminate the problem of determining the magnitude of the friction force $F_{\mu}$ when there is no motion $\dot{x}_{r}=0$. This corresponds to (2).

\subsubsection{Non-linear Spring Element}

The objective is to derive a non-linear spring element to be used in the implicit incremental NR scheme, which will mimic the behaviour of a friction force. The schematics and nomenclature of the spring are illustrated in Fig. 2.

The VWP for a general elastic body may be stated as $[23,22]$ :

$$
\begin{aligned}
\int_{V}\{\delta \varepsilon\}^{T}\{\sigma\} d V=\int_{A}\{\delta \mathbf{u}\}^{T}\{F\} d A+ & \int_{V}\{\delta \mathbf{u}\}^{T}\{\Phi\} d V \\
& +\sum_{i}\{\delta \mathbf{u}\}_{i}^{T}\{p\}_{i} .
\end{aligned}
$$

Assuming body forces negligible and writing the internal work as a summation over the elements and assuming the external forces are only applied in nodes (i.e.

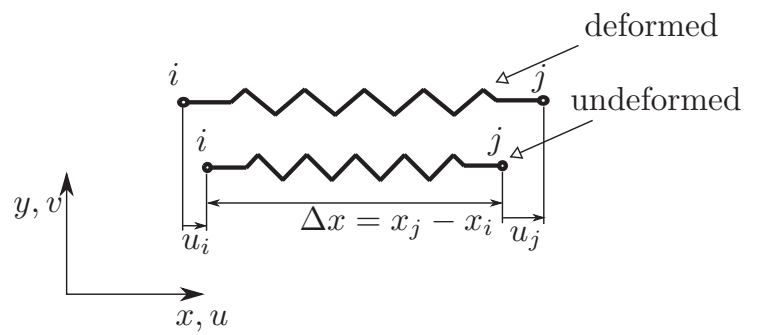

Figure 2: Deformed and undeformed one dimensional spring 
$\{\Phi\}=\{0\}$ and $\{F\}=\{0\})$ and given by the global force vector $\{P\}$, the VWP can be reduced to

$$
\sum_{e} \int_{V_{e}} \delta \varepsilon \sigma d V=\{\delta D\}^{T}\{P\}
$$

where displacements are described by the element nodal displacement vector $\{d\}$ or the global displacement vector $\{D\}$. Assuming the stress and strains are constant in each spring element, the integral on the left hand side of (4) can be evaluated as

$$
\sum_{e} \delta \varepsilon N^{e} L_{0}^{e}=\{\delta D\}^{T}\{P\}
$$

where the element forces are defined as $N^{e}=\varepsilon L_{0}^{e} k^{e}$ in which $k^{e}(\varepsilon)$ is a general non-linear stiffness dependent on the strain and $L_{0}^{e}$ is the initial length of the element $e$. The strain variations for each element are related to the displacement variations by

$$
\delta \varepsilon=\{\bar{B}\}^{T}\{\delta d\}
$$

where $\{\bar{B}\}$ is the strain-displacement vector. The straindisplacement vector can now be found by use of the Cauchy strain assumption $\varepsilon=\Delta L / L_{0}$. If the vertical and horizontal displacements of the two nodes, $i$ and $j$, (Fig. 2) are described by the vector

$$
\{d\}=\left\{\begin{array}{llll}
u_{i} & v_{i} & u_{j} & v_{j}
\end{array}\right\}^{T}
$$

then the strain in the spring element can be written as

$$
\varepsilon=\frac{L_{1}-L_{0}}{L_{0}}=\frac{\left(x_{j}-u_{j}\right)-\left(x_{i}-u_{i}\right)-\left(x_{j}-x_{i}\right)}{\left(x_{j}-x_{i}\right)}=\frac{\Delta u}{\Delta x}
$$

where $\Delta u=u_{j}-u_{i}$. By use of (7) and (8), the strain can now be written as

$$
\varepsilon=\{d\}^{T} \frac{1}{L_{0}}\left\{\begin{array}{llll}
-1 & 0 & 1 & 0
\end{array}\right\}^{T}=\{d\}^{T}\left\{B_{0}\right\}
$$

where the strain displacement vector $\left\{B_{0}\right\}$ is independent of the displacements and hence it is given the zero subscript. The variation in strain (6) then becomes $\delta \varepsilon=\{\delta d\}^{T}\left\{B_{0}\right\}$, which inserted into (5) gives

$$
\sum_{e}\{\delta d\}^{T}\left\{B_{0}\right\} N^{e} L_{0}^{e}=\{\delta D\}^{T}\{P\} .
$$

The VWP should hold for any virtual displacements which means that (10) reduces to

$$
\sum_{e}\left\{B_{0}\right\} N^{e} L_{0}^{e}=\{P\}
$$

which can be put on residual form as

$$
\{R\}=\left\{R_{\text {int }}\right\}-\left\{R_{\text {ext }}\right\}=\sum_{e}\left\{B_{0}\right\} N^{e} L_{0}^{e}-\{P\}=\{0\}
$$

and from the definition of the tangent stiffness matrix we have

$$
\begin{aligned}
{\left[K_{t}\right]=\frac{\partial\{R\}}{\partial\{D\}} } & =\sum_{e}\left\{B_{0}\right\} \frac{\partial N^{e}}{\partial \varepsilon} \frac{d \varepsilon}{\partial\{D\}} L_{0}^{e} \\
& =\sum_{e}\left\{B_{0}\right\}\left\{B_{0}\right\}^{T} L_{0}^{e} \frac{\partial N^{e}}{\partial \varepsilon}
\end{aligned}
$$

where $\partial N^{e} / \partial \varepsilon=L_{0}^{e} k^{e}(\varepsilon)$. Finally, the tangent stiffness matrix becomes

$$
\left[K_{t}\right]=\sum_{e}\left\{B_{0}\right\}\left\{B_{0}\right\}^{T} L_{0}^{e^{2}} k^{e}(\varepsilon) .
$$

\subsubsection{Choosing a Spring Stiffness Function}

From the definition of the strain-displacement vector (9), it is seen that the length of the spring $L_{0}^{e}$ cancels out from the tangent matrix (14). Therefore, it is convenient to redefine the non-linear element stiffness $k^{e}$ to be dependent on the displacement rather than the strain such that the length $L_{0}^{e}$ is eliminated in the element definition. A suitable element stiffness function is:

$$
k^{e}(\Delta u)=\frac{F_{n} \mu}{|\Delta u|+\varepsilon_{s}}
$$

where $\varepsilon_{s}$ is introduced to avoid zero division and to obtain a smoothing element force curve. The element stiffness and force curves are illustrated in Fig. 3.

Examining the element force curve $N^{e}(\Delta u)$ in Fig. 3, it is clear that the stiffness function (15) is a good choice as it produces a force curve very similar to that of a

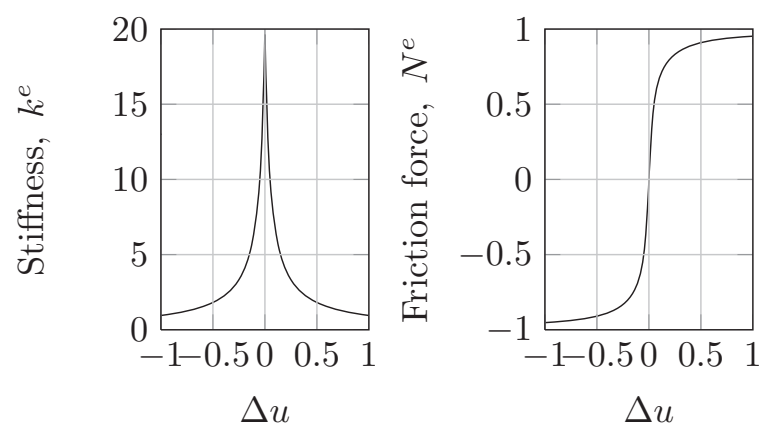

Figure 3: Element stiffness and force curves for $F_{n} \mu=1$ and $\varepsilon_{s}=$ 0.05 
friction force. The optimal value of $\varepsilon_{s}$ depends on the amount of movement in the sliding contact. For small movements $\varepsilon_{s}$ should be chosen small. Choosing too small values, the convergence of the incremental solver will be affected negatively and choosing too large values will affect the accuracy of the solution. A good choice (according to Fig. 3) is: $\varepsilon_{s} \approx \Delta u_{\max } / 100$.

The theory presented will enable the modelling of sliding friction for several contact points in an FE model. Independent of the sliding direction in each point, the resulting friction force will have the correct sign. However, this is under the assumption that the sliding does not change direction for $\Delta u \neq 0$. To assure the correct sign of the friction force a 'shift' is introduced such that

$$
k^{e}\left(\Delta u-\Delta u_{s}\right)=\frac{F_{n} \mu}{\left|\Delta u-\Delta u_{s}\right|+\varepsilon_{s}}
$$

where $\Delta u_{s}$ is set to $\Delta u$ in the event of changing sliding direction.

\subsubsection{Assumptions and Limitations}

The solution is quasi-static meaning that all frequency dependencies are discarded. The friction model is a Coulomb model and coefficients of friction are assumed constant and static and dynamic friction is equal.

\section{Theoretical Results - Validation}

In the following, bump foil strips with varying number of bumps are analysed using the numerical method, outlined in the previous section, and compared to analytical results. The geometry and nomenclature of the bump foils are illustrated in Fig. 4 and Tab. 1. For foil strips consisting of more than one bump, all bumps are given the same deflections.

Walowit and Anno [4] gave an analytical expression for the dimensionless deflection $\tilde{w}_{0}$, of the center position of a single bump, when subjected to a vertical load $W$. They assumed the bump ends free to rotate and move horizontally but restrained in vertical direction. This analytical expression is compared to results obtained from an equivalent FE model as illustrated in Fig. 5.

A mesh convergence study of the model showed, that sufficient accuracy may be obtained by having 8 layers of elements over the thickness and approximately 400 elements over the longitudinal direction.

The dimensionless deflection $\tilde{w}_{0}$, calculated analytically and numerically, is illustrated in Fig. 6 for different angular extends $\theta_{0}$ of the bump and for varying coefficients of friction $\mu$. Good agreement between the analytical and numerical results are observed for friction

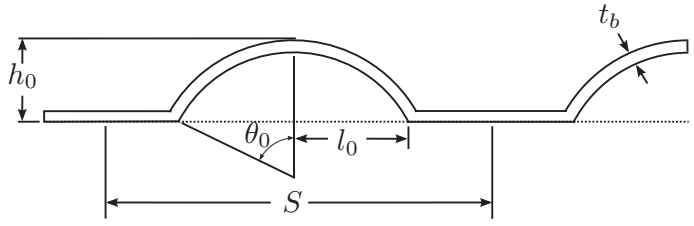

Figure 4: Bump foil geometry and nomenclature

Table 1: Geometry and material properties of the bump foil

\begin{tabular}{lr}
\hline Parameters & Values \\
\hline Bump foil thickness, $t_{b}$ & $0.127 \mathrm{~mm}$ \\
Bump foil height, $h_{0}$ & $0.9 \mathrm{~mm}$ \\
Bump foil pitch, $S$ & $7.00 \mathrm{~mm}$ \\
Bump half length, $l_{0}$ & $3.30 \mathrm{~mm}$ \\
Bump foil width, $w_{b}$ & $18 \mathrm{~mm}$ \\
Young's modulus of bump foil, $E$ & $2.07 \times 10^{11} \mathrm{~Pa}$ \\
Poisson's ratio of bump foil, $v$ & 0.3 \\
Coefficient of friction, $\mu$ & 0.20 \\
\hline
\end{tabular}

coefficients up to $\mu \approx 0.5$. Discrepancies begin to occur at $\mu>0.5$. However, the numerical analysis is not subjected to the same limiting assumptions as the analytical like e.g. longitudinal deflection correction [4].

Walowit and Anno [4] also derived an analytical expression for the foil flexibility which is commonly used together with 'the simple elastic foundation model' $[16,17]$. It is given as:

$$
Q \approx \frac{2 S}{E}\left(\frac{l_{0}}{t_{b}}\right)^{3}\left(1-v^{2}\right)
$$

and consequently, the stiffness per area is $K=1 / Q$. Comparing this stiffness to the results of the numerical

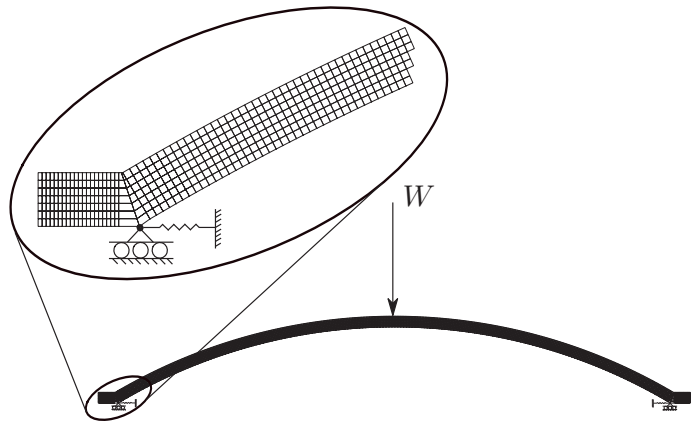

Figure 5: Finite element model and applied boundary conditions for numerical comparison to analytical results from Walowit and Anno [4] 


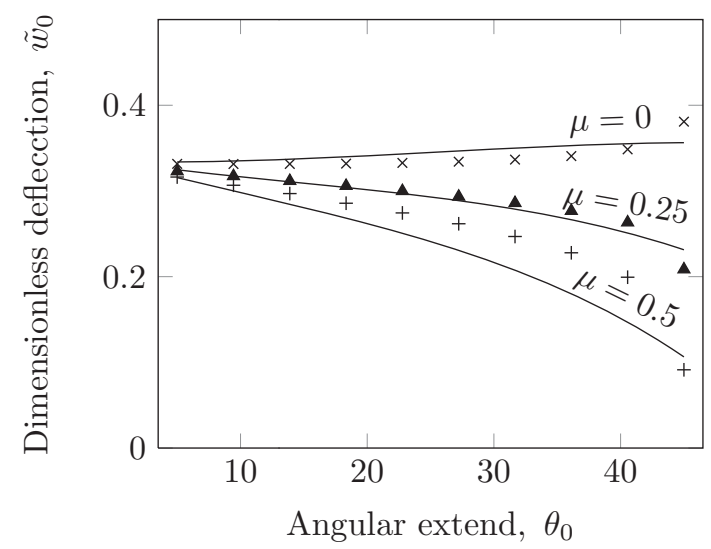

Figure 6: Single bump dimensionless deflection; analytical results (full lines) [4], numerical results (markers)

model of a single bump, as illustrated in Fig. 7, yields good agreement for varying coefficient of friction.

For the case of more than one bump and with the bump strip fixed in one end, the stiffness calculated numerically is diverging significantly from the analytical result [4]. This is illustrated in Fig. 8 and in accordance with e.g. $[1,5,15,16,17,18]$. The stiffness, predicted by the numerical procedure, is unequal during loading and unloading for $\mu \neq 0$. Fig. 8 is based on the loading process and the difference between loading and unloading is clearly illustrated in Fig. 9, which displays a load displacement diagram for $\mu=0.1$ and $\mu=0.2$. Here, a strip with four bumps is simulated by giving all bumps a gradual compression to approximately $25 \mu \mathrm{m}$ with small oscillations of $1.5 \mu \mathrm{m}$ amplitude occurring at approximately $5,10,15,20 \mu \mathrm{m}$ during the loading process. The particular bumpfoil geometry was designed for a journal bearing having a clearance of $50 \mu \mathrm{m}$, meaning that a compression of $25 \mu \mathrm{m}$ would result in a bearing eccentricity ratio of approximately 1.5 , which is a common value. The stiffness, related to the small 'local' hysteresis loops contained in the large 'global' hysteresis loop, is referred to as the local stiffness. It is found to be non-linear and significantly higher than the global. This is in good agreement with previous experimental studies performed by $\mathrm{Ku}$ and Heshmat [5].

The hysteresis loops cause the bump foil strip to provide Coulomb damping proportional to its confined area. The size of the confined area is dependent on where at the global hysteresis curve the deflection oscillation is taking place $(5,10,15$ or $20 \mu \mathrm{m})$. If the deflection is sufficiently large, the load versus displacement will track the global hysteresis curve during the unloading process. This situation is seen in Fig. 9a,

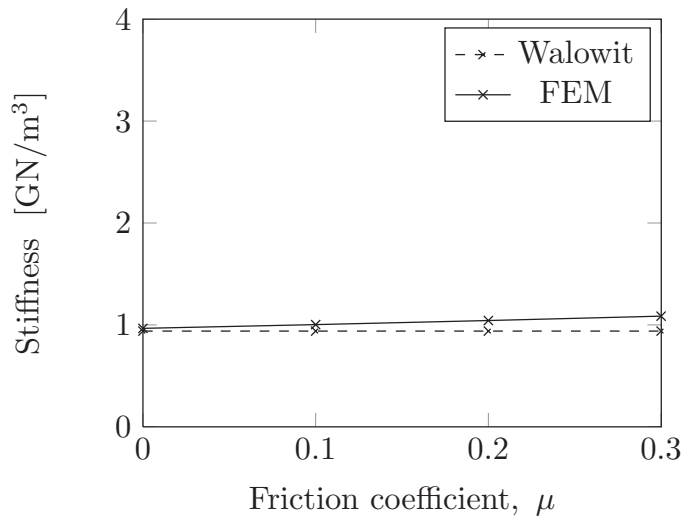

Figure 7: Stiffness of a single bump numerically calculated as function of varying coefficients of friction - comparison to analytical results of Walowit and Anno [4]

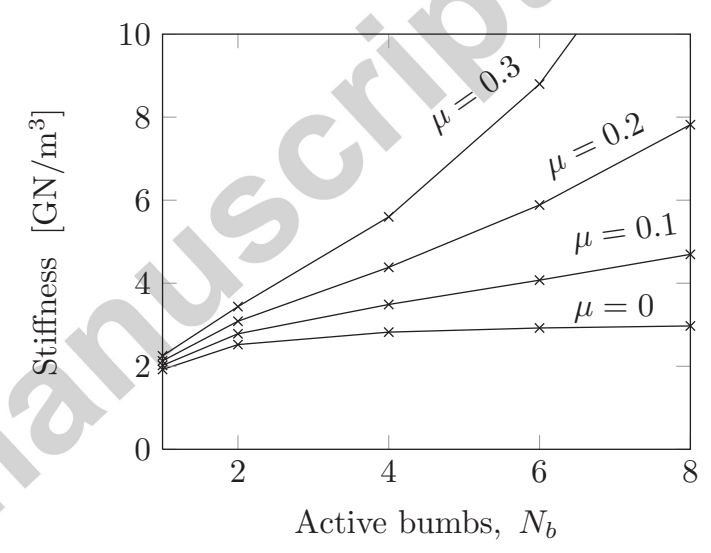

Figure 8: Stiffness calculated numerically - varying coefficients of friction and number of bumps in the foil strip

for the oscillation around $5 \mu \mathrm{m}$. In this case, the confined area grows significantly leading to more Coulomb damping, and the stiffness becomes highly non-linear, as it changes significantly at the points where the local load-displacement coincide with the global hysteresis loop. Tracking the global hysteresis curve corresponds to the situation where all contact points are sliding.

This can be seen in Fig. 10. Here a strip of four bumps, as illustrated in Fig. 10a, is subjected to displacement oscillations upon given a compression of $20 \mu \mathrm{m}$. The displacement oscillations are of amplitudes $1,3,5 \mu \mathrm{m}$ and the corresponding hysteresis curve is seen in Fig. 10b. In Fig. 10c, the friction forces versus the horizontal displacement for five selected contact points are illustrated. These hysteresis loops visually illustrates the amount of energy dissipation taking place in each of the selected contact points. For the low amplitude of $1 \mu \mathrm{m}$ (green line), it is clear that the movement 
(a)

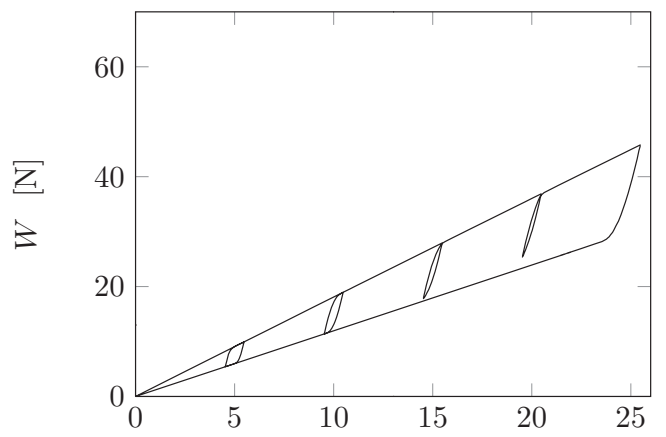

(b)

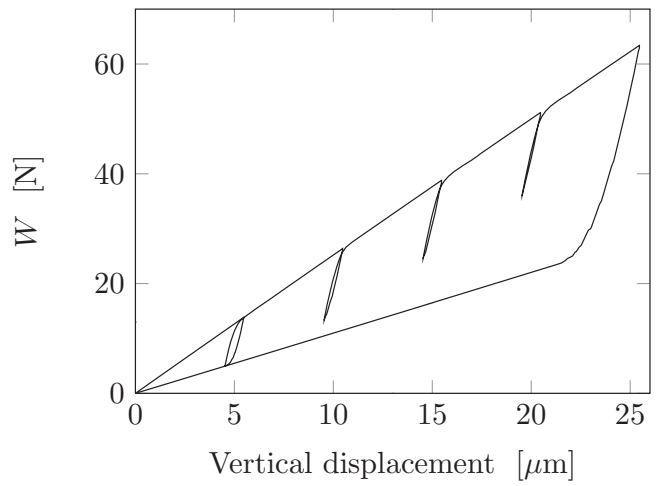

Figure 9: Theoretical results of a bump strip, given a global compression of approximately $25 \mu \mathrm{m}$ with local oscillations of $1.5 \mu \mathrm{m}$ amplitude occurring at approximately 5, 10, 15,20 $\mu \mathrm{m}$ during the loading process. (a) Using a coefficient of friction $\mu=0.1$ (b) Using a coefficient of friction $\mu=0.2$

in sliding points \#1 through \#5 is zero, meaning that energy is only dissipated in the contact points \#6, \#7, \#8. For the higher amplitudes of $3 \mu \mathrm{m}$ and $5 \mu \mathrm{m}$, (red and blue lines respectively) all the points are sliding when the local load-displacement curves (Fig. 10b) tracks the global curve.

It is important to highlight how a relatively small increase in the load amplitude, from $15 \mathrm{~N}$ to $20 \mathrm{~N}$, will increase the energy dissipation by approximately 10 times and at the same time, the energy dissipation only doubles for an amplitude increase from $20 \mathrm{~N}$ to $25 \mathrm{~N}$. This is a consequence of the left most bumps being pinned for the lowest load amplitudes, and it illustrates the importance of the bump geometry and friction properties in terms of maximizing the energy dissipation. For instance, the dissipated energy would have been much higher, for an amplitude of $15 \mathrm{~N}$, if the coefficient of friction had been lower, since this would have prevented bumps from being pinned. (a)

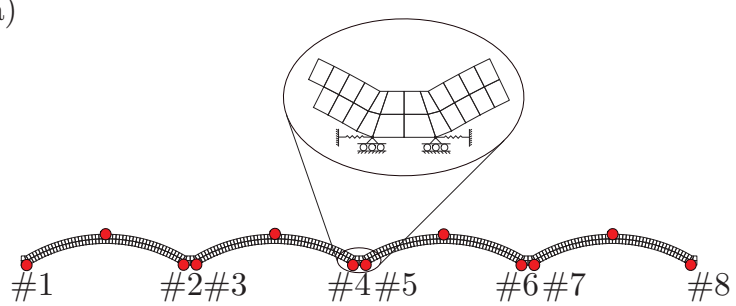

(b)

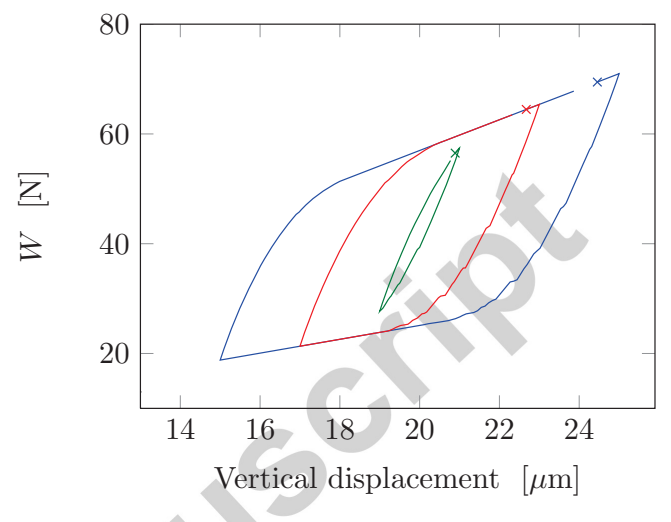

(c)

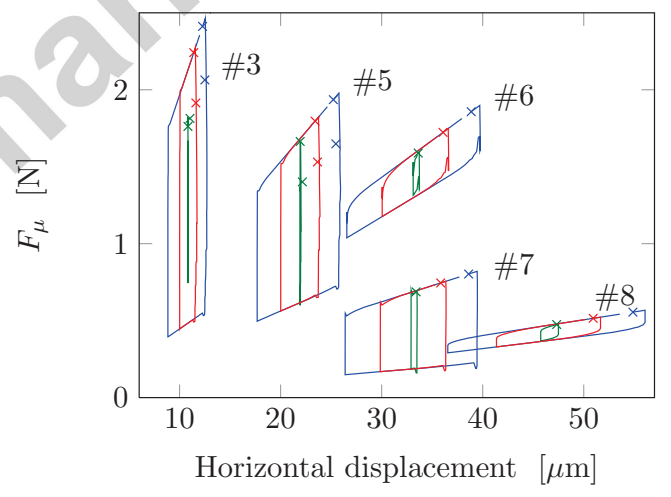

Figure 10: (a) Bump foil strip. Contact points including friction marked with a red dot. The foil thickness is magnified for illustration purpose. (b) Local hysteresis curves for different load amplitudes. (c) Friction force versus horizontal deflection in selected contact points.

\section{Experimental Results}

In order to validate the implemented numerical model of the bump foil, its results are compared to experimental results. In this section, the focus is set on the static as well as the dynamic behaviour of the bump foil. The data is obtained using an experimental test rig at the Technical University of Denmark (DTU), designed and constructed specifically for this purpose. 


\subsection{Experimental Setup at DTU}

The test rig used for characterizing the bump foil behaviour can be seen in Fig. 11. This test rig enables to study the static and dynamic characteristics of the bump foils.

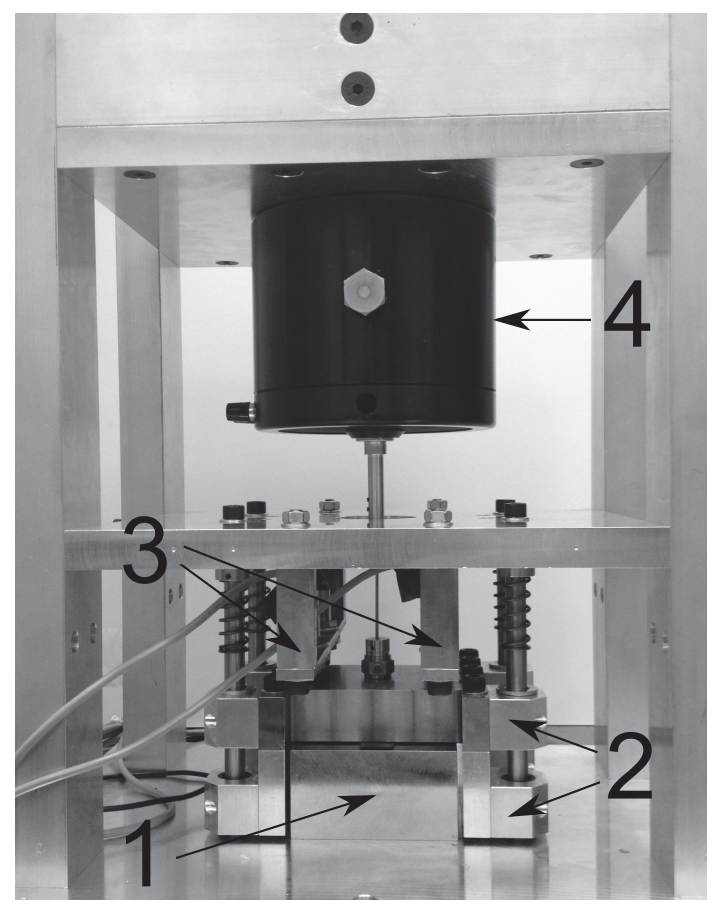

Figure 11: Test setup for characterizing the static and dynamic properties of the bump foil

The core of the setup is composed by two steel blocks (FE 510 D, ISO 630), labelled number 1 in Fig. 11. The upper block features linear ball bearings that follow four vertical guiding rods, see number 2 in Fig 11. Two bearings are mating with each guiding rod. This arrangement enables the upper block to move vertically minimizing tilting motion, while the lower block is fixed to the base. The tested foil strip is placed in between the parallel mating surfaces of these blocks. One of the foil strip ends is clamped, and the other one is free.

The arrangement enables to relate directly the vertical displacement of the upper block with the deflections of the bumps of the foil strip tested. The displacements are measured using three displacement probes looking at the upper surface of the moving block, see number 3 in Fig. 11. The sensors are located in a 'triangle' arrangement, to detect if any undesired tilting motion is taking place during the experimental tests. The upper block can be loaded statically or dynamically, in order to induce deflections of the foil strip placed underneath. Static load is applied by means of calibrated weights, whereas dynamic load is obtained by using an electromagnetic shaker, a steel stinger with a diameter of $2 \mathrm{~mm}$ and a piezoelectric load cell, see number 4 in Fig. 11.

\subsection{Static Results}

The test rig was used to obtain experimental results regarding the relationship between applied static load and resulting deflection of the bump foil. The geometry and material properties of the tested foil are listed in Tab. 1 and the foil material is Inconel X750 hardened for maximum yield stress. A foil specimen originally consisting of 10 bumps was progressively shortened down to $8,6,4$ and 2 bumps. For each configuration, five full load cycles (loading and unloading) were performed. The standard deviation of the measured deflections was calculated, in order to check the influence of random errors over the results. The largest uncertainty interval obtained is 8 microns and the lowest one is 2 microns. The uncertainty intervals are not included in the figures to avoid overcrowding.

The results obtained with strips of two and four bumps are compared to theoretical results and illustrated in Fig. 12 and the results of a strip with six bumps is illustrated in Fig. 13.

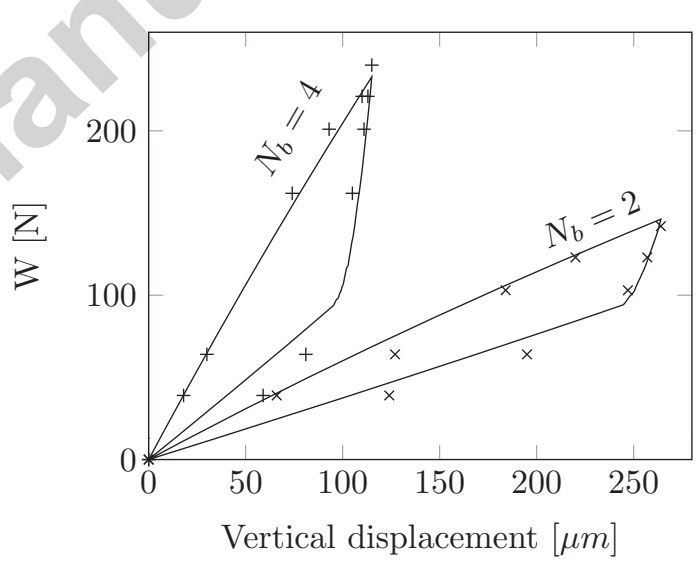

Figure 12: Hysteresis loops for two and four bump strips; numerical results using $\mu=0.2$ (full lines), experimental results (markers)

Good agreement between the experimental and theoretical results are found when using a coefficient of friction $\mu=0.2$ for the simulations. This value corresponds well with common values which is typical in the range $0.1<\mu<0.5$ for steel against steel ( 0.5 in vacuum) and also with the results obtained by e.g [5, 12]. For strips with higher number of bumps, higher global stiffness and larger discrepancies with theoretical results are observed. The results obtained for a strip with 6 bumps, see Fig. 13, portray these trends. The discrepancies can be attributed to geometrical imperfections of 


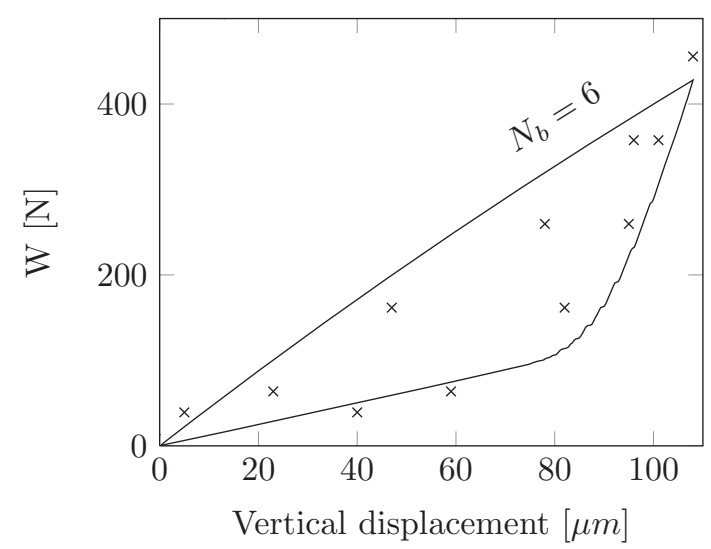

Figure 13: Hysteresis loops for a six bump strip; numerical results using $\mu=0.2$ (full lines), experimental results (markers)

the foils, specifically different bump heights, that entail that not all bumps are in contact with the mating surface from the beginning of the loading cycle. This effect becomes more relevant for higher number of bumps. Similar trends are observed when testing strips of 8 and 10 bumps.

\subsection{Dynamic Results}

The next set of experimental results deal with the effect of applied load frequency over the hysteresis curves. A static preload and a dynamic load are simultaneously applied on the foil strip. The preload was adjusted to $40 \mathrm{~N}$ and $90 \mathrm{~N}$, in order to study the effect of this parameter over the obtained results. Regarding the dynamic load, a sine wave of fixed amplitude and frequency was fed into the electromagnetic shaker in order to induce the foil deflections. The amplitude of the dynamic load was tuned to obtain different displacement amplitudes for the hysteresis cycles. Hence, results for 2 (blue), 4 (red), 8 (green), 12 (black) microns of displacement amplitude are obtained.

The reported applied force over the foil specimen is determined as the summation of the preload, the value measured by the piezoelectric load cell associated with the shaker stinger, plus the inertia force coming from the upper steel block, quantified using a piezoelectric accelerometer. The deflections are measured using the displacement probes. The reported hysteresis curves are obtained by averaging the loading cycles obtained over a one minute long test. Repeatability was checked by repeating the test five times, with different foil specimens, obtaining similar results. Variability of the results was on the same order of magnitude than the one registered for the static testing.
In order to check for the influence of the test rig arrangement on the measured hysteresis curves, the foil specimen was replaced with a coil spring. Assuming that the damping contribution from this element is negligible, the setup enabled to determine the baseline damping coming from the test rig. Up to $60 \mathrm{~Hz}$ of excitation frequency, such effect was found to be negligible, compared with the energy dissipation observed when the foil specimen was tested.

The first set of results compare the static and dynamic testing results obtained for a four bump foil strip, see Fig. 14. The static results are obtained by loading the foil using calibrated weights, whereas the dynamic ones correspond to a preload of $40 \mathrm{~N}$ and a dynamic loading frequency of $1 \mathrm{~Hz}$. The amplitude of the dynamic loading is tuned to obtain four different displacement amplitudes. Although the results obtained with a loading frequency of $1 \mathrm{~Hz}$ do not have relevance from the practical point of view, they do enable to establish a direct link between the static results shown in the previous section and the dynamic ones presented here.

In Fig. 14, the local hysteresis curves follow an almost purely harmonic motion for small displacement amplitude, and they are placed inside the global loop. However, when the displacement amplitude surpasses a threshold value, they start to track the global hysteresis curve. For that condition, two stages can be easily recognized in both the loading and unloading path of the cycle, characterized by two different slopes for the curve. These results are qualitatively coincident with the results from the quasistatic theoretical model shown before in Fig. 10b, regarding the dependence of the hysteresis loop shape on the motion amplitude. According to the theoretical model, the 'high slope' behaviour can be explained by the fact that some bumps of the strip are sticking, hence the stiffness is dominated by elastic deformation of the bumps. When they start to slide, the hysteresis path switches to a 'low slope' behaviour, where the stiffness is dominated by the friction forces.

Since the local hysteresis curves are amplitude dependant, both the stiffness and damping properties are strongly influenced by it. Even though for small motion amplitude it could be possible to assume that the resulting foil displacement is harmonic, once the local curve hits the global one a highly non-linear motion is achieved. This behaviour could have significant effects when calculating an equivalent linearised damping coefficient based on the energy dissipated during one local hysteresis cycle, since such analysis is based on assuming pure harmonic motion for the load displacement curve.

In order to check for loading frequency dependency 


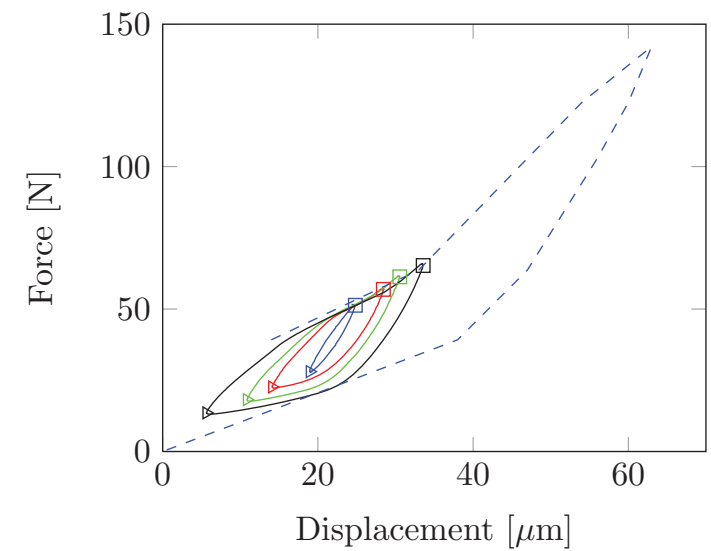

Figure 14: Comparison of the static (dashed blue line) and dynamic testing results, for different displacement amplitudes (2 (blue), 4 (red), 8 (green), 12 (black) microns). The applied load frequency is $1 \mathrm{~Hz}$. The preload is $40 \mathrm{~N}$, and the foil strip contains 4 bumps.

of the observed local hysteresis curves, the results for a strip containing 3 bumps were obtained. Two different preloads are applied, and the loading frequency is set to $1 \mathrm{~Hz}, 10 \mathrm{~Hz}, 20 \mathrm{~Hz}, 40 \mathrm{~Hz}$ respectively. The loading frequencies tested here are well below the first resonant frequency of the setup, which is around $100 \mathrm{~Hz}$. Although the studied frequency range might seem quite limited when compared to the broad frequency range in which an industrial bump foil bearing operates, a distinctive modification in the overall behavior of the hysteresis loops is already observed within the studied range. Furthermore, the maximum frequency for perfoming the dynamic testing is limited by the natural frequency of the setup and by the influence of the baseline damping generated by the test rig itself, as discussed previously.

The results obtained for a loading frequency of $1 \mathrm{~Hz}$, see Fig. 15, are coincident with the ones reported before for the 4 bumps strip, see Fig. 14. By increasing the loading frequency, significant changes in the hysteresis behaviour are observed. This is especially true at the larger displacement amplitudes, as it can be seen in Fig. 16, 17, 18. A direct comparison between the results for $1 \mathrm{~Hz}$ and $40 \mathrm{~Hz}$ can be seen in Fig. 19. For higher loading frequency, the area enclosed by the local hysteresis curves tends to become smaller, and it seems that they are not tracing the global static one any more. Closer inspection reveals that the 'high slope' behaviour observed for the low frequency results tends to diminish or disappear for excitations with a higher frequency. Following the reasoning established before, this could be attributed to the fact that all the bumps exhibit slid-

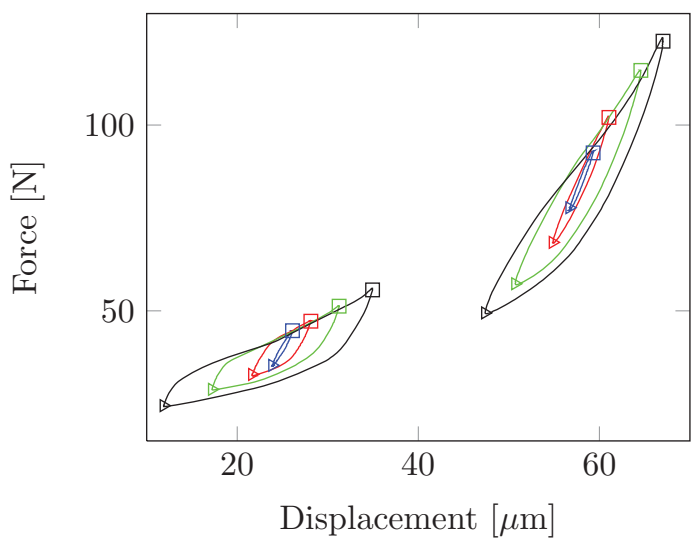

Figure 15: Results of the dynamic testing, for different displacement amplitudes (2 (blue), 4 (red), 8 (green), 12 (black) microns). The applied load frequency is $1 \mathrm{~Hz}$. The preloads are $40 \mathrm{~N}$ and $90 \mathrm{~N}$. The foil strip contains 3 bumps.

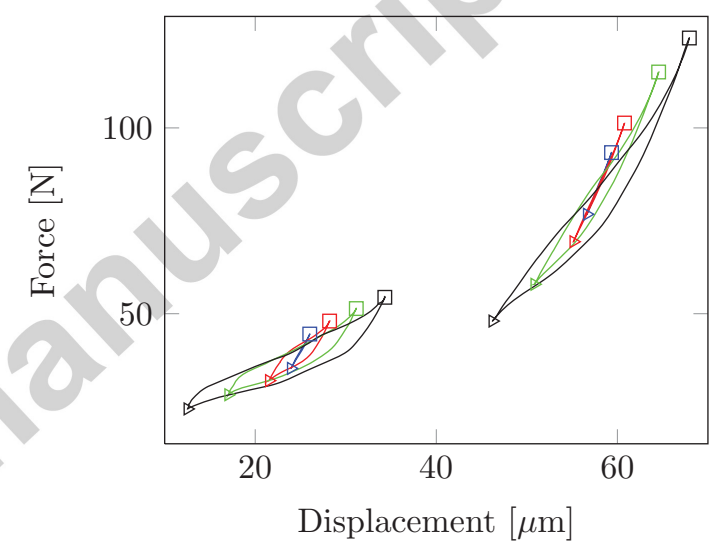

Figure 16: Results of the dynamic testing, for different displacement amplitudes (2 (blue), 4 (red), 8 (green), 12 (black) microns). The applied load frequency is $10 \mathrm{~Hz}$. The preloads are $40 \mathrm{~N}$ and $90 \mathrm{~N}$. The foil strip contains 3 bumps.

ing motion, without switching to a sticking phase when the direction of the displacement is inverted. The comparison depicted in Fig. 19 shows clearly this trend.

It can be observed that the end points for the loaddisplacement curves for the low and high frequency test are the same. The mathematical model showed that this would not be the case if the friction coefficient was reduced as can be seen in Fig. 9a and Fig. 9b. This indicates, that a reduction of the constant coefficient of friction alone, can not explain the 'flattening' of the hysteresis curves.

One could argue that the bumps inertia forces could play a role in the observed behaviour, however the maximum acceleration measured in the vertical direction is around $1.5-2.0 \mathrm{~m} / \mathrm{s}^{2}$. Assuming the entire mass of the 


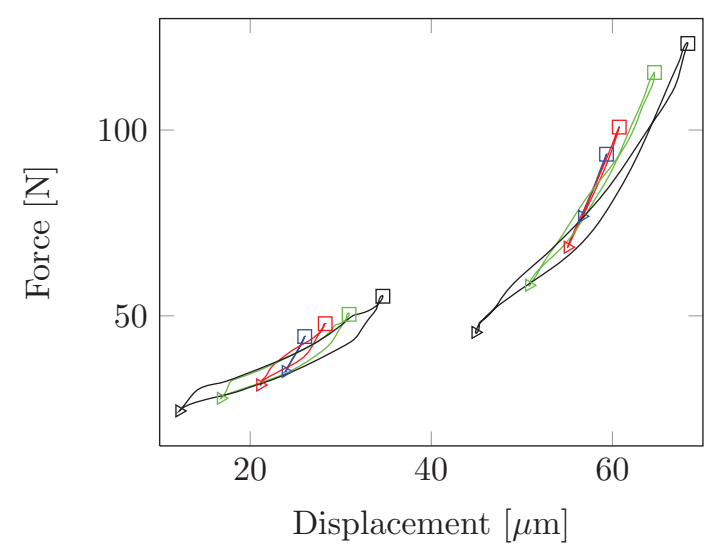

Figure 17: Results of the dynamic testing, for different displacement amplitudes (2 (blue), 4 (red), 8 (green), 12 (black) microns). The applied load frequency is $20 \mathrm{~Hz}$. The preloads are $40 \mathrm{~N}$ and $90 \mathrm{~N}$. The foil strip contains 3 bumps.

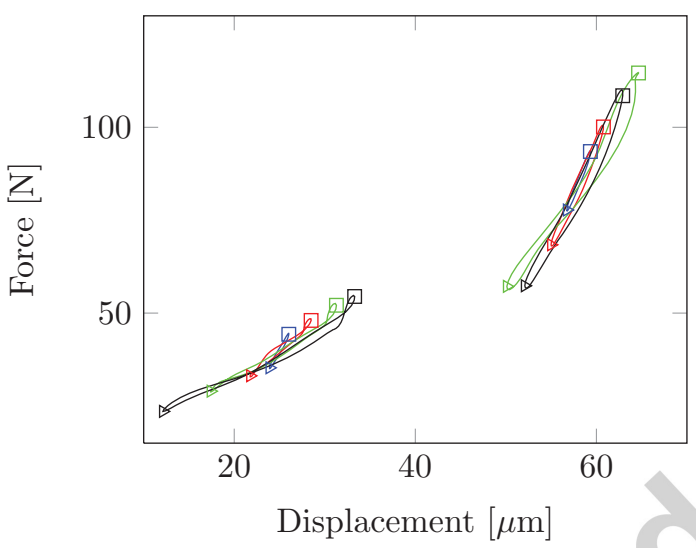

Figure 18: Results of the dynamic testing, for different displacement amplitudes (2 (blue), 4 (red), 8 (green), 12 (black) microns). The applied load frequency is $40 \mathrm{~Hz}$. The preloads are $40 \mathrm{~N}$ and $90 \mathrm{~N}$. The foil strip contains 3 bumps.

foil specimen, which is $0.45 \mathrm{~g}$, to be concentrated in one sliding point and multiplying it with the horizontal acceleration of this point would yield a very small force. In fact a force with an order $F_{\mu} / 1000$, if comparing to the numerical results illustrated in Fig. 10.

The apparent reduction of the bump sticking phase for higher excitation frequencies could be attributed to a momentary modification of the friction coefficient in or around the transition between sliding and sticking. An acceleration $\ddot{x}_{r}$ dependant friction coefficient was described as early as 1943 by Sampson et al. [24]. They showed that the dry friction coefficient would decrease in the acceleration stage of the slip and remain low during the deceleration. Later investigations by Sakamoto [25] confirmed this phenomenon. The friction coeffi-

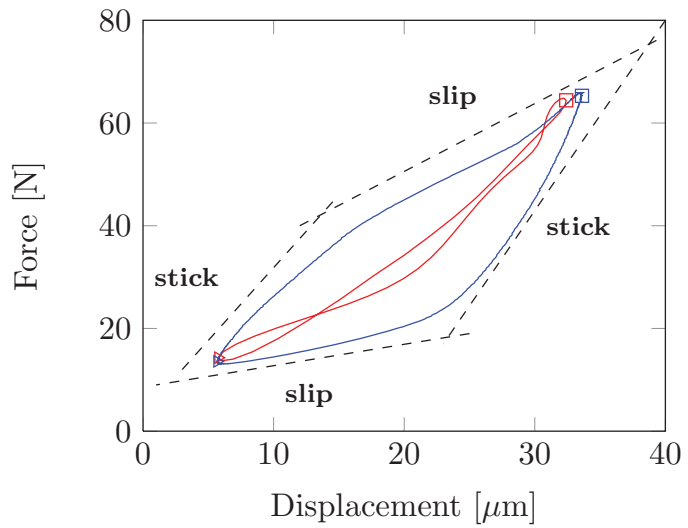

Figure 19: Comparison of results for the dynamic testing: local hysteresis loop measured for loading frequency $1 \mathrm{~Hz}$ (blue) and $40 \mathrm{~Hz}$ (red). The foil strip contains 3 bumps and the preload is $40 \mathrm{~N}$.

cient was obtained experimentally to $\mu \approx 0.4$ in the beginning of the slip and dropped significantly to $\mu \approx 0.1$ over the acceleration stage where after it remained constant. Their experiments did not deal with the transition between the stick and slip phase. However, the results may still serve to explain why the global hysteresis curve become narrower at increasing frequency i.e. higher accelerations close to the transition between stick and slip.

Other literature in the field of tribology addresses the effect of vibration in the normal direction of two mating surfaces over the friction coefficient. Chowdhury and Helali [26] performed an experiment regarding this issue. A clear trend is observed, regarding the reduction of the friction coefficient with both the amplitude and the frequency of the normal vibration, for different tested materials. These authors relate the observed trend to an eventual reduction of the effective contact area between the mating surfaces when vibrations are taking place. This effect may also be relevant for the bump foil strip, as the normal loads in the contact points is oscillating.

\section{Conclusion and Future Aspects}

In this article, a theoretical and experimental study has been carried out, aimed at investigating the behaviour of bump foils used in compliant gas bearings. The investigation has focused on the static and dynamic behaviour of a bump foil pressed between two rigid parallel surfaces. A quasi-static non-linear finite element model of the complete foil geometry has been developed, including the effects of the foil flexibility and the friction forces in the contact points. An original 
approach for modelling the friction forces was implemented, based on the usage of non-linear spring elements. The results from the numerical model regarding load-displacement behaviour have been compared with theoretical and experimental results coming from the literature, as well as experimental data obtained from a dedicated test rig.

The numerical model was compared to previously published analytical results [4]. For the stiffness of a single bump (free-free) good agreement between analytical and numerical results were observed at different coefficients of friction. The analytically calculated stiffness is commonly used in combination with the 'simple elastic foundation model'. However, for a strip consisting of several bumps (fixed-free), the numerical model indicated that the analytical method significantly underestimates the stiffness, hence the 'simple elastic foundation model' is generally underestimating the contribution of the structural stiffness.

The hysteresis loops obtained numerically, corresponded well with the experimentally obtained hysteresis loops for low load frequencies $<5 \mathrm{~Hz}$ and both stiffness and damping was found to be highly non-linear. The numerical model was able to reproduce both the global load-displacement curves as well as the local ones, considering smaller vertical displacements around an equilibrium position. Two distinctive patterns of motion were observed for the local hysteresis loop based on the theoretical and experimental results. If the vertical displacement perturbations are small enough, then the hysteresis loop tends to follow a sinusoidal motion, where the dominant effect corresponds to the bumps flexibility since most of the bumps are pinned due to friction forces. If the displacement surpasses a threshold value, then the local hysteresis loop tracks the global one, exhibiting two distinctive slopes associated with the dominance of the bumps elastic forces or the sliding friction forces respectively. In this condition, the transition towards sliding friction behaviour greatly enhances the energy dissipation properties of the foil, and the resulting vertical displacements deviates significantly from the purely harmonic motion.

The experimental determination of the local hysteresis curves for higher loading frequencies revealed that both the stiffness and equivalent damping properties of the bump are strongly load frequency dependant. At higher frequencies, the experimental results deviates significantly from the theoretical as the hysteresis loops tend to 'flatten' and the energy dissipated per load cycle reduces significantly. This phenomenon tends to alter the stiffness such that it becomes less non-linear but still of the same approximate magnitude. For small load amplitudes and high frequency, the motion of the bump deflection is nearly harmonic, but for larger load amplitudes the motion is still non-harmonic, even though less distorted compared to the low frequency case.

The 'flattening' of the hysteresis loops at high frequency seems to be caused by the absence of the stick phase i.e. the foil contacts are operating constantly in the slip phase. The authors are under the impression, that inertia effects will be small and that the phenomenon may as well be related to instantaneous variations in the coefficient of friction or other local contact phenomena.

In order to improve the numerical model to a state of accuracy desired in the design of foil bearings, the effect of the load frequency needs to be taken into account. In that regard, more research needs to be conducted to properly understand the 'flattening' phenomenon of the hysteresis curves experimentally obtained and documented in this work. This future work can be divided in the following steps:

- the effect of inertia forces needs to be investigated by including mass in the numerical model and perform a time integration analysis.

- Previous published results [24, 25, 26] has proven significant friction variations due to vibrations in normal and perpendicular direction of the contact points as well a significant dependency of sliding acceleration. These and related effects needs to be studied in more details.

\section{Appendix A. Iterative solution based on the Newton-Raphson method}

In the implicit incremental Newton-Raphson (NR) scheme, the load is applied in $n$ increments, for each of which the displacement $D^{n}$ is found iteratively by satisfying the non-linear equilibrium condition which can be written in residual form as:

$$
R\left(D^{n}\right)=R_{\text {int }}\left(D^{n}\right)-P^{n} .
$$

If $D_{i}^{n}$ is an approximate solution to the exact solution $D^{n}$, then a first order Taylor expansion gives an equilibrium equation for the next NR-step as

$$
R\left(D_{i+1}^{n}\right) \approx R\left(D_{i}^{n}\right)+\frac{d R\left(D_{i}^{n}\right)}{d D} \Delta D_{i}^{n}=0 .
$$

If we now define the tangent as 


$$
K_{t} \equiv \frac{d R\left(D_{i}^{n}\right)}{d D}
$$

then the equilibrium equation (A.2) can be written as

$$
K_{t} \Delta D_{i}^{n}=-R\left(D_{i}^{n}\right)
$$

or inserting (A.1)

$$
K_{t} \Delta D_{i}^{n}=-R_{\text {int }}\left(D_{i}^{n}\right)+P^{n} .
$$

When the equilibrium equation (A.5) has been solved the displacements are updated from

$$
D_{i+1}^{n}=D_{i}^{n}+\Delta D_{i}^{n} .
$$

The tangent is then updated with the new displacement $D_{i}^{n}=D_{i+1}^{n}$ and the procedure is repeated until the norm of the residual is sufficiently small. Here, the NR method was derived for a scalar problem, but it is directly applicable to vector problems.

\section{References}

[1] C.-P. R. Ku, H. Heshmat, Compliant foil bearing structural stiffness analysis: Part i--theoretical model including strip and variable bump foil geometry, Journal of Tribology 114 (1992) 394 400.

[2] C.-P. R. Ku, H. Heshmat, Structural stiffness and coulomb damping in compliant foil journal bearings: theoretical considerations, Tribology transactions 37 (1994) 525-533.

[3] C.-P. R. Ku, H. Heshmat, Structural stiffness and coulomb damping in compliant foil journal bearings: parametric studies, Tribology transactions 37 (1994) 455-462.

[4] J. A. Walowit, J. N. Anno, Modern developments in lubrication mechanics, Applied Science Publishers London, 1975.

[5] C.-P. R. Ku, H. Heshmat, Compliant foil bearing structural stiffness analysis. ii: Experimental investigation, Journal of tribology 115 (1993) 364-369.

[6] J. P. Peng, M. Carpino, Coulomb friction damping effects in elastically supported gas foil bearings, Tribology transactions 37 (1994) 91-98.

[7] H. Heshmat, C. P. Ku, Structural damping of self-acting compliant foil journal bearings, Journal of tribology 116 (1994) 76-82.

[8] C.-P. R. Ku, H. Heshmat, Effects of static load on dynamic structural properties in a flexible supported foil journal bearing, ASME Transactions Journal of Vibration Acoustics 116 (1994) 257-262.

[9] D. Rubio, L. San Andrés, Bump-type foil bearing structural stiffness: Experiments and predictions, ASME Conference Proceedings 2004 (2004) 671-679.

[10] D. Rubio, L. San Andrés, Bump-type foil bearing structural stiffness: experiments and predictions, Journal of Engineering for Gas Turbines and Power(Transactions of the ASME) 128 (2006) 653-660.

[11] I. Iordanoff, Analysis of an aerodynamic compliant foil thrust bearing: method for a rapid design, Journal of tribology 121 (1999) 816-822.

[12] L. Rubio, D.and San Andrés, Structural stiffness, dry friction coefficient, and equivalent viscous damping in a bump-type foil gas bearing, Journal of engineering for gas turbines and power 129 (2007) 494-502.
[13] M. Salehi, H. Heshmat, J. F. Walton, On the frictional damping characterization of compliant bump foils, Journal of tribology 125 (2003) 804-813.

[14] S. Le Lez, M. Arghir, J. Frene, A new bump-type foil bearing structure analytical model, Journal of engineering for gas turbines and power 129 (2007) 1047-1057.

[15] S. Le Lez, M. Arghir, J. Frene, A dynamic model for dissipative structures used in bump-type foil bearings, Tribology Transactions 52 (2008) 36-46.

[16] H. Heshmat, J. A. Walowit, O. Pinkus, Analysis of gas lubricated compliant thrust bearings, Journal of Lubrication Technology 105 (1983) 638-646.

[17] H. Heshmat, J. A. Walowit, O. Pinkus, Analysis of gaslubricated foil journal bearings, Journal of Lubrication Technology 105 (1983) 647-655.

[18] D. Lee, Y.-C. Kim, K.-W. Kim, The dynamic performance analysis of foil journal bearings considering coulomb friction: Rotating unbalance response, Tribology Transactions 52 (2009) 146-156.

[19] G. Zywica, The static performace analysis of the foil bearing structure, acta mechanica et automatica 5 (2011).

[20] G. Zywica, The dynamic performance analysis of the foil bearing structure, Acta mechanica et automatica 7 (2013).

[21] Z. Kozanecki, J. Kicinski, G. Zywica, Numerical model of the high speed rotors supported on variable geometry bearings, in: IUTAM Symposium on Emerging Trends in Rotor Dynamics, 2011, pp. 217-227.

[22] S. Krenk, Non-linear modeling and analysis of solids and structures, Cambridge University Press, 2009.

[23] R. D. Cook, D. S. Malkus, M. E. Plesha, J. W. Witt, Concepts and applications of finite element analysis, 4 ed., John Wiley, New York, 2002.

[24] J. B. Sampson, F. Morgan, D. W. Reed, M. Muskat, Studies in lubrication: Xii. friction behavior during the slip portion of the stick-slip process, Journal of Applied Physics 14 (1943) 689700 .

[25] T. Sakamoto, Normal displacement and dynamic friction characteristics in a stick-slip process, Tribology international 20 (1987) 25-31.

[26] M. A. Chowdhury, M. M. Helali, The effect of frequency of vibration and humidity on the coefficient of friction, Tribology International 39 (2006) 958-962. 